\title{
PREVENTION OF SEPSIS IN HANDS AFFECTED BY LEPROSY
}

\section{A. S. GarretT}

Area Superintendent, Onitsha Province, East Nigeria

The majority of serious septic troubles in the hands and feet in leprosy usually start as trival injuries.

As a method of combatting these in their earliest stages, I thought of issuing to those with trophic changes something in the nature of an " Iodine pencil," but using a more modem antiseptic.

Messrs. Ciba kindly let me have a sample of their plastic nose-sprays together with some "Bradosol" as the antiseptic. I have issued about 40 to patients with a tendency to trophic lesions at a time of year (the beginning of the farming season) when injuries are most common. All patients had recent evidence of injuries which would benefit by prompt attention.

There has been a noticeable absence of sepsis in all these patients and they unitedly say that small injuries heal quicker than they did previously. 